clinicians. Methods: The American Health Information Community (AHIC) is a federal advisory body organized to make recommendations to the Secretary of the US Department of Health and Human Services on how to accelerate the adoption of HIT. In July 2007, AHIC advanced recommendations to improve electronic health information exchange via the implementation of standards for FH information. The first step to developing interoperable HIT systems for the collection, interpretation, and exchange of $\mathrm{FH}$ information is the development of a widely accepted core minimum dataset for FH. To this end, the Personalized Healthcare Workgroup of the AHIC assembled the FH Multi-Stakeholder Workgroup to develop a minimum core dataset. The workgroup is comprised of over 40 members representing approximately 18 different public and private organizations with expertise in the area of $\mathrm{FH}$, HIT, and healthcare delivery. Results: The FH core dataset for primary care was developed using an interactive, iterative process that used the proposed standards for FH information contained in the Continuity of Care Record (CCR) as a foundational document. Some key features of the core dataset are, FH information should be collected in a structured manner that permits the generation of a pedigree, age of disease onset should be collected, and selfidentified race and ethnicity data should be included as FH information. The document outlining the requirements will be made available for public comment in early 2008. Conclusions: The document should serve as a solid foundation for public and private efforts to create HIT systems and demonstration projects examining the utility of interoperable $\mathrm{FH}$ information in clinical and research settings.

\section{Abstract C-D2-05 \\ Creation of State Legislation to Protect and Facilitate Use and Exchange of Electronic Health Information}

Margaret J. Gunter, PhD, Lovelace Clinic Foundation; Shelley Carter, RN, MPH, MCRP, Lovelace Clinic Foundation; Bob Mayer, New Mexico Department of Health; Randall McDonald, JD, Miller Stratvert P.A.

Background: In examining potential barriers to implementing a statewide health information exchange in NM, we identified as a key barrier NM's laws regarding the protection of health information. The laws are fragmented and dated, adopted prior to HIPAA, and address only health data in paper-based formats. Enactment of specific state legislation authorizing transmission, use, and exchange of electronic health information would eliminate the detrimental effects of the existing state laws. Aim: To develop and pass comprehensive state privacy legislation in New Mexico governing the use, storage, and exchange of electronic health information. Methods: Review of health privacy legislation nationally, interviews with privacy experts, development of a draft of the legislation, and solicitation of input from a variety of stakeholder groups to identify controversial areas of the legislation and to garner legislative support were performed. Key privacy experts (e.g., legislators, healthcare providers, and attorneys) discussed their concerns and opinions about electronic transmission of health information. Attorneys researched privacy legislation in other states and drafted NM's Electronic Health Information Act. This draft was examined and discussed with numerous stakeholder groups to determine needed revisions. Results: Interviews with key privacy experts and stakeholders revealed conflicting points of view concerning the legislation, especially regarding issues of patient consent. Patient privacy advocates strongly favored patient control of access to their health data and more restrictive rules for patient consent than required by HIPAA. In contrast, providers, payers, and advocates for health information exchange networks typically favored less restrictive patient consent (consistent with current HIPAA regulations) to avoid additional time, burden, expense, and delays in implementing health information exchange. Review of legislation in other states revealed only Minnesota and Nevada have enacted laws specifically addressing electronic health information exchange. Conclusions: Current NM legislation (and legislation in most other states) does not adequately address the use and exchange of electronic health information. Current efforts to develop health information exchange networks in NM and elsewhere require resolution of key patient privacy issues and the passage of appropriate legislation. Funded by the Agency for Healthcare Research and Quality.

\section{Health Economics}

\section{Abstract C-C4-01 \\ Prevalence-Based Costs of HIV-Positive Patients With Multiple HAART Switches}

Richard T. Meenan, PhD, MPH, MBA, Kaiser Permanente Center for Health Research; Michael A. Horberg, MD, MAS, FACP, Division of Research, Kaiser Permanente Northern California; Maureen C. O'Keeffe-Rosetti, MS, Kaiser Permanente Center for Health Research; Terry Kimes, MS, Kaiser Permanente Center for Health Research; Wendy Leyden, MS, Division of Research, Kaiser Permanente Northern California; Diana Antoniskis, MD, Immune Deficiency Clinic, Kaiser Permanente Northwest

Background/Aims: Highly active antiretroviral therapy (HAART) successfully treats patients with HIV. However, many patients do not achieve complete suppression. Perhaps the most important reason for treatment failure is infection with antiretroviral drug-resistant variants, which threatens to increase treatment cost as well as limit HAART's clinical effectiveness. This study examines the resource utilization and cost implications of multi-drug resistance using the HIV+ population of Kaiser Permanente Northern California (KPNC). Methods: Retrospective cohort analysis of all HIV+ patients in KPNC between January 2000 and June 2005 (total study period). Continuous active membership of 12 months and minimum 12 months of continuous HAART. Cases defined as HIV+ patients on third or later HAART regimen; controls defined as HIV+ patients on first or second HAART regimen. Regimen switch defined as combination change of two or more additions or subtractions of antiretroviral therapy (ART) drugs to or from an existing HAART regimen. Costs include outpatient, inpatient, and pharmacy categories. Results: All results are for 2004 only (291 cases, 1,676 controls); costs reported in person-years. Mean total pharmacy costs were $\$ 17,668$ for cases vs $\$ 12,417$ for controls $(P<0.04)$. Of mean pharmacy costs for cases, $85 \%$ were ART-based (controls $=90 \%$ ). Mean number of 2004 fills for both ART and non-ART drugs were higher for cases (20 vs 15 for ART; 21 vs 15 for non-ART). Mean total outpatient costs were $\$ 3,275$ for cases vs $\$ 2,238$ for controls $(P<0.06)$. Mean ER visits and mean primary care visits, but not specialty care visits, were both higher for cases than for controls $(P<0.03)$. Mean inpatient costs were $\$ 3,803$ for cases vs $\$ 1,738$ for controls. Mean number of stays was higher for cases than for controls ( 0.29 vs 0.16 ; $P<0.05$ ), but length of stay did not differ between groups. Conclusions: In 2004, patients with HIV on at least their third HAART regimen incurred mean pharmacy costs $40 \%$ higher than similar patients on first or second regimen. Outpatient and inpatient costs also appear higher among cases, but not strikingly so. Overall cost differentials between cases and controls may be underestimates, as laboratory, radiology, and home health have not yet been added. These cost categories may well widen the cost differences between HIV+ patients who have been forced to switch HAART regimens more than once relative to those who have not.

\section{Abstract C-C4-04 \\ Entering and Exiting the Coverage Gap by Medicare Part D Beneficiaries With Diabetes: a TRIAD Study}

Julie A. Schmittdiel, $\mathrm{PhD}$, Kaiser Permanente Northern California Division of Research; Susan L. Ettner, PhD, University of California Los Angeles; Vicki Fung, PhD, Kaiser Permanente Northern California Division of Research; Jie Huang, PhD, Kaiser Permanente Northern California Division of Research; Norman Turk, MS, University of California Los Angeles; Elaine Quiter, MS, University of California Los Angeles; Bix Swain, MS, Kaiser Permanente Northern California Division of Research; John Hsu, MD, MBA, MSCE, Kaiser Permanente Northern California Division of Research; Carol M. Mangione, MD MPH, University of California Los Angeles

Background/Aims: Many Medicare Part D enrollees face gaps in their prescription drug cost coverage after $\$ 2,250$ in annual drug expenditures. Beneficiaries with chronic illnesses may be particularly vulnerable to coverage gaps, with estimates ranging as high as $64 \%$ of diabetic beneficiaries entering the gap. We examined total drug costs and out-of-pocket drug costs, and rates of entry and exit into the Part D coverage gap for beneficiaries with diabetes in two large California Medicare Advantage plans. Methods: Beneficiaries from two large health plans in the TRIAD (Translating Research Into Action for Diabetes) Study offering Medicare Advantage Part 
D plans in California were included if they had diabetes; signed up for Part D between November 15, 2005 and March 1, 2006; and had a drug coverage gap starting at $\$ 2,250$. Entry and exit into the gap was determined using pharmacy databases. Beneficiaries hit the gap after incurring $\$ 2,250$ in total drug costs; beneficiaries exited the gap after incurring $\$ 3,600$ in out-ofpocket costs. Results: In 2006, 25\% of the 45,732 beneficiaries reached the coverage gap and then paid the full price of their drugs. Only $1 \%$ of beneficiaries exited the gap and qualified for 'catastrophic' drug cost assistance. On average, diabetes beneficiaries incurred $\$ 1,942$ in total drug costs during 2006. For beneficiaries who did not enter the coverage gap, total drug expenditures and out-of-pocket drug expenditures remained stable over the year. For the beneficiaries who fell into the drug coverage gap in 2006, total drug costs were much higher overall and decreased over the year as outof-pocket expenses increased. Conclusions: This is the first examination of diabetic Medicare beneficiaries under the Part D program. Fewer entered the coverage gap than had been previously estimated. Those entering the gap, however, had lower subsequent monthly drug expenditures. Potential reasons for both include lower than expected drug prices or drug adherence and higher than expected use of less expensive drugs, such as generics. Future work will examine these alternative explanations and explore risk factors for entering the Part D coverage gap.

\section{Cardiovascular Disease}

Abstract C-D1-02

Disparities in Patients' Ability to Participate in a Web-Based Intervention to Improve Blood Pressure Control

Beverly B. Green, MD, MPH, Group Health Center for Health Studies; James D. Ralston, MD, MPH, Group Health Center for Health Studies; David Carrell, PhD, Group Health Center for Health Studies; Lynda Tyll, RN, MS, Group Health Center for Health Studies; Andrea Cook, PhD, Group Health Center for Health Studies

Background: Electronic medical records and Web communications bring new opportunities to provide healthcare. Still unknown is whether this type of care will improve health outcomes and its affect on utilization and costs. Care in this environment may also be limited by the 'digital divide' between users and nonusers of Web communications. 'Electronic Communications and Home Blood Pressure Monitoring (e-BP)' is a randomized controlled trial designed to test the effectiveness of home BP monitoring, Web communications (secure patient e-mail that interfaces with an electronic medical record shared between patients and providers), and pharmaceutical care to improve hypertension control. We describe the proportions and characteristics of hypertensive patients willing and able to participate in an electronic healthcare intervention. Methods: Patients with uncomplicated hypertension received mailed invitations, a telephone survey, and in-person visits to determine their eligibility and willingness to participate. Eligibility requirements included access to a computer, the Internet, and an e-mail address. We performed a cross-sectional study on the subset willing to answer the telephone survey $(\mathrm{n}=7286)$, evaluating demographic differences between three populations: (1) those ineligible due to at least one computer access reason; (2) those eligible based on computer access, but not enrolled in the study for other reasons; and (3) those eligible and enrolled. We ran Pearson's chi-square test to evaluate bivariate associations. All $P$-values were two-sided. Results: Of those answering the telephone survey, $78 \%$ had computer, Internet, and e-mail access. These patients were significantly more likely to be male $(P<0.001)$, younger $(P<0.001)$, Caucasian $(P=0.01)$, more educated $(P<0.001)$, employed $(P<0.001)$, and with a commercial healthcare plan $(P<0.001)$, but no more likely to have a home BP machine. Enrolled patients with computer access and uncontrolled hypertension were not more likely to be male, but otherwise they were similar to those with computer access but not enrolled for other reasons. Conclusions: Most patients we contacted with hypertension had computer, Internet, and e-mail access, but a 'digital divide' persists. Chronic disease disproportionately affects older people, racial minorities, and those with less education. If electronic healthcare is to be effective in improving chronic disease health outcomes, additional efforts will be required to include these patients.
Abstract C-D1-03

Atrial Flutter is an Independent Predictor of Increased Mortality During Long Term Follow-Up

Ravi K. Mareedu, MD, Marshfield Clinic; Kodlipet C. Dharmashankar, MD, Marshfield Clinic; Ihab B. Abdalrahman, MD, Marshfield Clinic; Robert T. Greenlee, PhD, Marshfield Clinic Research Foundation; Po-Huang Chyou, $\mathrm{PhD}$, Marshfield Clinic Research Foundation; Param P. Sharma, MD, Marshfield Clinic; John J. Hayes, MD, Marshfield Clinic; Peter N. Smith, MD, Marshfield Clinic; Humberto J. Vidaillet, MD, Marshfield Clinic Research Foundation and Marshfield Clinic

Background: Atrial flutter (Afltr) and atrial fibrillation (Afib) are the two cardiac arrhythmias most frequently encountered in medical practice. Unlike Afib for which epidemiologic data paved the way for clinical trials that subsequently revolutionized its treatment, little is known about the course of the estimated 200,000 new cases diagnosed with Afltr in the U.S. every year. The purpose of this investigation was to determine whether Afltr is an independent predictor of death. Methods: Using the Marshfield Epidemiologic Study Area, a database that captures nearly all medical care and deaths among its 58,820 residents, we identified all patients newly diagnosed with Afltr in the region from July 1, 1991, through June 30, 1995. Incident cases were age and gender matched with controls and all were followed prospectively. To minimize the potential contribution of associated comorbidities, the analysis was limited to incident Afltr cases without Afib and adjusted for 11 pre-existing risk factors selected a priori; age, gender, stroke, myocardial infarction, heart failure, hypertension, rheumatic heart disease, hyperlipidemia, chronic pulmonary disease, diabetes mellitus and thyroid abnormality. Multivariate Cox models were fitted to obtain hazard ratios and $95 \%$ confidence intervals. Results: A total of 1356 person-years of follow-up were completed in 76 patients and 76 controls. There were 54 deaths $(71 \%)$ among Afltr group and 37 deaths (49\%) among controls $(P=0.0079)$. After adjustment, the risk of death among incident cases with Afltr was 1.5 times that of controls (Hazard Ratio $=1.56$; 95\% Confidence Interval: 1.01-2.41, $P=0.04$ ). Conclusions: (1) Our data suggest that Afltr is an independent predictor of death. (2) Since Afltr can now be easily and safely cured in the outpatient setting in virtually all patients, if our findings can be reproduced in other populations, consideration should be given to conducting prospective randomized trials to determine whether eliminating Afltr can improve survival in these individuals.

\section{IRB}

\section{Abstract C-D4-01}

IRB Review of Multi-Institutional Research

Jeffrey P. Braff, DrPH, CIP, Kaiser Foundation Research Institute; Sarah M. Greene, MPH, Group Health Cooperative; Robert J. Reid, MD, Group Health Cooperative

Statement of the Problem: Because IRB review of multi-institutional research can involve as many IRBs as there are institutions participating in the research, the process can be frustrating, time-consuming, and unnecessarily iterative for both IRBs and researchers, and can also possibly cause researchers to miss funding opportunities. Definition of the Intended Outcome: Among a network of 16 affiliated large organizations engaged in significant epidemiologic research, each with its own IRB, arrive at a method to improve the IRB review process of multi-institutional research, while at the same time, assuring that regulatory and policy requirements are met. Description of Program: Determine which group of low-risk data-only studies will be used to beta test the new approaches to IRB review of inter-institutional research. Communicate with involved researchers, IRB Chairs, and IRB Administrators to discuss the review process options to determine the approaches to be tested. Evaluate the processes used for the review of each study and modify process as appropriate for the next study. Inform Chairs and Administrators of the results of each test. Recommend an approach for adoption by all members of the network. Evaluation Method: Testing of procedures currently underway. Suggestions: When completed, this process may prove adaptable to others engaged in multi-institutional research review. 